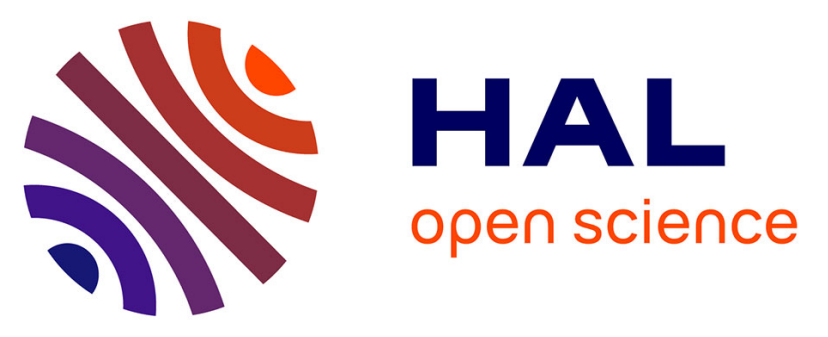

\title{
Quantification of the methylation at the GNAS locus identifies subtypes of sporadic pseudohypoparathyroidism type Ib
}

Stéphanie Maupetit-Méhouas, Virginie Mariot, Christelle Reynes, Gyulène Bertrand, François Feuillet, Jean-Claude Carel, Dominique Simon, Hélène Bihan, Vincent Gajdos, Eve Devouge, et al.

\section{To cite this version:}

Stéphanie Maupetit-Méhouas, Virginie Mariot, Christelle Reynes, Gyulène Bertrand, François Feuillet, et al.. Quantification of the methylation at the GNAS locus identifies subtypes of sporadic pseudohypoparathyroidism type Ib. Journal of Medical Genetics, 2010, 48 (1), pp.55. 10.1136/jmg.2010.081356 . hal-00579025

\section{HAL Id: hal-00579025 \\ https://hal.science/hal-00579025}

Submitted on 23 Mar 2011

HAL is a multi-disciplinary open access archive for the deposit and dissemination of scientific research documents, whether they are published or not. The documents may come from teaching and research institutions in France or abroad, or from public or private research centers.
L'archive ouverte pluridisciplinaire HAL, est destinée au dépôt et à la diffusion de documents scientifiques de niveau recherche, publiés ou non, émanant des établissements d'enseignement et de recherche français ou étrangers, des laboratoires publics ou privés. 


\section{Quantification of the methylation at the GNAS locus identifies subtypes of sporadic pseudohypoparathyroidism type Ib}

Stéphanie Maupetit-Méhouas ${ }^{1}$, Virginie Mariot $^{1}$, Christelle Reynes ${ }^{2}$, Guylène Bertrand ${ }^{13}$, Francois Feillet $^{3}$, Jean-Claude Carel $^{4}$, Dominique Simon ${ }^{4}$, Hélène Bihan ${ }^{5}$, Vincent Gajdos ${ }^{6}$, Eve Devouge Savitha Shenoy ${ }^{8}$, Placide Agbo-Kpati ${ }^{9}$, Anne Ronan ${ }^{10}$, Catherine Naud ${ }^{11}$, Anne Lienhardt ${ }^{12}$, Caroline Silve ${ }^{1,13}$, Agnès Linglart ${ }^{1,14}$

${ }^{1}$ INSERM ; Université Paris Descartes. U561, Hôpital Saint Vincent de Paul, 75014 Paris, France

${ }^{2}$ MTi-Université Paris Diderot. INSERM U973, Rue Hélène Brion, 75013 Paris, France

${ }^{3}$ Centre de référence des maladies héréditaires du métabolisme. INSERM U954, CHU Brabois Enfant, 54500 Vandoeuvre les Nancy, France

${ }^{4}$ Assistance Publique-Hôpitaux de Paris ; Université Paris 7. Department of pediatric endocrinology and diabetology, Robert Debré Hospital, 75019 Paris, France

${ }^{5}$ Assistance Publique-Hôpitaux de Paris. Endocrinologie Diabétologie et maladies métaboliques, Hôpital Avicennes, 93000 Bobigny, France

${ }^{6}$ Assistance Publique-Hôpitaux de Paris. Service de pédiatrie, hôpital Beclère, Clamart, France

${ }^{7}$ Service de pédiatrie, hôpital d'Arras, France

${ }^{8}$ Leicester Royal Infirmary. Leicester LE1 5WW, UK

${ }^{9}$ Service de pédiatrie, hôpital de Lagny, Lagny, France

${ }^{10}$ Hunter Genetics Unit. Waratah, PO Box 84, NSW 2298, Australia

${ }^{11}$ Service de pédiatrie Bretagne sud, 56100 Lorient, France

${ }^{12}$ Centre de référence des maladies rares du métabolisme du calcium et du phosphore. Service de pédiatrie, Hôpital de la mère et de l'enfant, 87000 Limoges, France

${ }^{13}$ Assistance Publique-Hôpitaux de Paris ; Université Paris 7. Hôpital Bichat Claude Bernard, Service de Biochimie hormonale et génétique, 75018 Paris, France 
${ }^{14}$ Assistance Publique-Hôpitaux de Paris. Endocrinologie-diabétologie pédiatrique et Centre de référence des maladies rares du métabolisme du calcium et du phosphore, Hôpital St-Vincent de Paul, 75014 Paris, France

KEY TERMS: pseudohypoparathyroidism, quantitative methylation, subtypes, somatic mosaicism

Word count : 4362 words in the manuscript

Correspondence should be addressed to Agnès Linglart: agnes.linglart@svp.aphp.fr

Telephone : +3314048 8401 ; Fax number : +3314048 8340

INSERM U986, Hôpital Saint Vincent de Paul, 82 avenue Denfert-Rochereau, 75014 Paris, France 


\section{ABSTRACT}

BACKGROUND: Pseudohypoparathyroidism type $\mathrm{Ib}$ (PHP-Ib) is due to epigenetic changes at the imprinted GNAS locus including loss of methylation at the A/B differentially methylated region (DMR) and sometimes at the XL and AS DMRs and gain of methylation at the NESP DMR.

Objective: To investigate if quantitative measurement of the methylation at the GNAS DMRs identifies subtypes of PHP-Ib.

DESIGN AND METHODS: In 19 patients with PHP-Ib and 7 controls, methylation was characterized at the four GNAS DMRs through combined bisulfite restriction analysis and quantified through cytosine-specific real-time PCR in blood lymphocyte DNA.

Results: A principal component analysis using the percent of methylation at 7 cytosines of the GNAS locus provided three clusters of subjects (controls $n=7$, autosomal dominant PHP-Ib with loss of methylation restricted to the A/B DMR $\mathrm{n}=3$ and sporadic PHP-Ib with broad GNAS methylation changes $n=16$ ) that matched perfectly the combined bisulfite restriction analysis classification. Furthermore, we identified three sub-clusters of patients with sporadic PHP-Ib that displayed different patterns of methylation: incomplete changes at all DMRs compatible with somatic mosaicism $(n=5)$, profound epigenetic changes at all DMRs $(n=8)$, and unmodified methylation at XL in contrast with the other DMRs (n=3). Interestingly, PTH level at the time of diagnosis correlated with the percent of methylation at the $\mathrm{A} / \mathrm{B} \mathrm{DMR}$.

Conclusion: Quantitative assessment of the methylation in blood lymphocyte DNA is of clinical relevance, allows the diagnosis of PHP-Ib, and identifies subtypes of PHP-Ib. Our epigenetic findings suggest mosaicism at least in some patients.

KEY TERMS: pseudohypoparathyroidism, quantitative methylation, subtypes, somatic mosaicism 


\section{INTRODUCTION}

Parental imprinting refers to mechanisms that lead to the repression of gene expression from one parental allele. Genes subjected to parental imprinting are usually clustered in regions rich in $\mathrm{CpG}$ dinucleotides and contain imprinting control elements in differentially methylated regions (DMRs). In most loci, the parent-specific expressed transcripts are associated with a pattern of non or low methylated DNA, whereas the non expressed transcripts are associated with a pattern of methylated DNA (1).

GNAS is an imprinted locus that produces several transcripts comprising $\mathrm{G}_{\mathrm{s}} \alpha$, the alpha stimulatory subunit of the G-protein, XL, A/B (also referred as 1A), NESP and the antisense transcript AS. Due to differential methylation of their promoters, most transcripts originate from one parental allele only. $\mathrm{XL}, \mathrm{A} / \mathrm{B}$ and AS are transcribed from the paternal allele ; NESP is transcribed from the maternal allele only (2) (3). The promoter of $G_{s} \alpha$ is not differentially methylated and therefore, $G_{s} \alpha$ expression arises from both alleles in most tissues (Figure 1). However, due to a yet incompletely understood imprinting mechanism, $G_{s} \alpha$ is expressed from the maternal allele only in several tissues including the renal proximal tubule, the thyroid, the pituitary and the gonads (4) (5) (6).

Pseudohypoparathyroidism type Ib (PHP-Ib) is a rare disease characterized by the development of hormonal resistance comprising PTH resistance and mild TSH resistance (7). Affected patients display no or mild features of Albright hereditary osteodystrophy (obesity, short stature, ectopic ossifications and bone shape abnormalities), i.e. findings that were initially described in pseudohypoparathyroidism type Ia (PHP-Ia) (8) (9) (10). In contrast to patients with PHP-Ia who carry heterozygous loss of function mutations of the $\mathrm{G}_{\mathrm{s}} \alpha$ coding sequence (11) (12), patients with PHP-Ib do not have mutation in the coding sequence of $\mathrm{G}_{\mathrm{s}} \alpha$, but present with epigenetic changes at one or several of the promoters of the GNAS locus (13). 
Based on the extent of these epigenetic changes, PHP-Ib has now been divided in different categories. In affected patients, the loss of methylation at the maternal A/B DMR of GNAS is associated with decreased $\mathrm{G}_{\mathrm{s}} \alpha$ expression in imprinted tissues and PTH resistance. In the autosomal dominant form of PHP-Ib (AD-PHP-Ib) caused in most patients by a maternally inherited 3-kb deletion within the coding sequence of the STX16 gene (14), and in one family by a maternally inherited 4.3-kb overlapping the $3-\mathrm{kb}$ deletion by $1.2-\mathrm{kb}$ (15). Affected individuals show a loss of methylation restricted to the maternal A/B DMR of GNAS. Both deletions remove an imprinting control element (ICE) located $\approx 220 \mathrm{~kb}$ upstream of the exon A/B of GNAS (Figure 1). The paternal transmission of those deletions does not affect the methylation of the A/B DMR of GNAS, nor $\mathrm{G}_{\mathrm{s}} \alpha$ expression (14) (15). In addition to those deletions within the STX16 gene, two deletions removing an ICE close to the AS and NESP DMRs have been described in three families so far (16) (17). In addition to the loss of methylation at the A/B DMR, the maternal transmission of these deletions is associated with methylation changes at other DMRs of GNAS on the maternal allele, i.e. loss of methylation at XL and AS, gain of methylation at NESP. Besides familial PHP-Ib, most patients are the unique affected individual of their family and thereafter considered as sporadic (sporPHP-Ib). The patients affected with sporPHP-Ib usually show broad methylation changes at their maternal GNAS locus. Although the loss of methylation at the A/B DMR of GNAS is constant in affected patients and confirms the diagnosis of PHP-Ib, the methylation changes have been described by us and others as uneven at the AS, XL and NESP DMRs (13) (18) (19) (9) (20).

We propose that the different epigenetic patterns observed in patients with sporPHP-Ib likely reflect different underlying causes of the disease. In order to test our hypothesis, we quantified and compared the methylation of cytosines at four DMRs of GNAS in patients affected with AD-PHP-Ib, sporPHP-Ib and controls. The epigenetic patterns were then correlated to the clinical and biochemical features of the patients. 


\section{PATIENTS AND METHODS}

\section{Patients}

Seven controls and 19 subjects affected with PHP-Ib were analyzed. Five patients, comprising one (PHP1) affected with AD-PHP-Ib and carrying a maternal STX16 deletion, and four affected with sporPHP-Ib (PHP4, PHP14, PHP15 and PHP17) have been previously reported in (19) (9).

When available, age at diagnosis, features of Albright osteodystrophy (height, body mass index, ectopic ossification, brachydactyly) and hormonal resistance (calcemia, phosphatemia, serum level of PTH, TSH, 25-OH vitamin D) were recorded retrospectively from medical files. Because the diagnosis of the disease was made in different hospitals, the laboratory techniques used to measure calcium, phosphorus, PTH, TSH and 25-OH vitamin D levels are different. Except for one patient (PHP1) diagnosed more than twenty years ago, all PTH assays were immunometric and measured the intact PTH. Clinical and biochemical characteristics of the patients at diagnosis (before any treatment) are provided in Table 1. Magnesium levels were available for 2 patients only, therefore are not provided here. All patients gave their informed consent for the genetic and epigenetic studies. For the present report, characterization of the methylation through combined bisulfite restriction analysis and quantification of the methylation at the four DMRs of GNAS were performed in all patients, including those previously reported.

\section{Detection of the STX16 deletion, exclusion of a deletion within NESP and analysis of the methylation at the four DMRs of GNAS}

The search for the common 3-kb deletion within STX16 and the determination of the methylation pattern of the GNAS locus through digestion of PCR amplified DNA after bisulfite treatment (combined bisulfite restriction analysis) were performed as previously described (9) except that A/B and AS DMRs were each amplified using a single PCR.

In order to exclude a monoallelic deletion removing the NESP exon, we amplified one target sequence of the NESP exon located in the genomic sequence that is removed by the two deletions described in some patients with PHP-Ib (16) through quantitative real-time PCR. We compared the results to that 
of the amplification of a single-copy gene MYH9. This approach was first validated in controls by comparing amplification of two target sequences within the NESP exon and two reference genes (MYH9 and RB1) as performed by Soufir and coll. (21). Primers are provided in Supplementary Table 1.

The quantification of the methylation was adapted from Thomassin et al. (22). Genomic DNA was treated with sodium bisulfite in agarose beads (23), amplified by PCR and analyzed by real-time PCR using primers specific for differentially methylated cytosines. For each cytosine analyzed, two primer sets were designed using the oligo6 software : a non discriminative (ND) primer pair allowing the quantification of the sum of methylated and unmethylated DNA and a discriminative (D) primer pair allowing the quantification of the methylated PCR products only (Supplementary Figure 1). This D primer is designed so that its most 3' nucleotide is a locked nucleic acid (LNA) and is complementary to the cytosine analyzed. The presence of the LNA at the 3' end of the D primer ensures an efficient discrimination between methylated and unmethylated DNA. Real-time amplification was performed using $2 \mu \mathrm{L}$ of PCR product diluted at $1 / 10^{4}$ as reported in Supplementary Table 1 using the LightCycler® 480 SYBR Green I Master Mix (Roche Applied Bioscience). For each DMR, experiments were performed in duplicates on two different cytosines except for NESP (only one cytosine due to the impossibility to identify accurate primers for an additional cytosine). Three DNAs were included in each experiment and served as internal controls to ensure repeatability. When measured onto two distinct bisulfite conversions of controls DNA, the quantification of the methylation provided similar results. For each experiment, linear correlations $\left(r^{2}>0.90\right.$ and $\left.p<0.05\right)$ between theoretical and measured percent of methylation were built using mixtures of PCR products obtained from amplification of bisulfite treated methylated and unmethylated DMR matrices inserted in TOPO-TA cloning plasmids (Invitrogen, Cergy-Pontoise, France). The standard curves were constructed using an increment of 5 between 0 and $40 \%$ of methylated DNA, and an increment of 10 between 40 to $100 \%$ of methylated DNA (Supplementary Figure 1, panel b). The slope and yintercept of these linear correlations were used to normalize the percent of measured methylation (see below). At a given cytosine, the percent of measured methylation was calculated using the following equations: 
Percent of measured methylation $=\left[100 \mathrm{x}\left(1+\mathrm{E}^{\mathrm{ND}}\right)^{\mathrm{CtND}} /\left(1+\mathrm{E}^{\mathrm{D}}\right)^{\mathrm{CtD}}\right]-$ background, in which

Background $=100 /(1+\mathrm{E})^{\Delta \mathrm{Ct}(\mathrm{UnMT}-\mathrm{MT})}$

where $\mathrm{E}$ is efficacy of amplification, $\mathrm{Ct}$ is cycle threshold, UnMT and MT are totally unmethylated and methylated DNA matrices, respectively.

The results are presented as percent of methylation normalized using the equation:

Percent of methylation $=\operatorname{slope}_{(\text {linear correlation })} \mathrm{x}$ percent of measured methylation $+\mathrm{y}$-intercept ${ }_{(\text {linear }}$ correlation)

The percent of methylation measured at two distinct cytosines of each DMR (A/B, XL and AS) were highly correlated (Supplementary Figure 2). Therefore values are provided throughout the manuscript and figures for one representative cytosine (AB2 for A/B, AS2 for AS, XL1 for XL and NE for NESP) except if mentioned otherwise. Except for XL2, the cytosines studied by real-time PCR were different from those analyzed by the combined bisulfite restriction analysis (Supplementary Figure 1, panel a).

The quantification of the methylation at the A/B DMR of GNAS was confirmed using pyrosequencing (methods in Supplementary data).

\section{Statistical analysis}

Quantitative measures of the patients characteristics (clinical and biochemical) are presented as median \pm standard error (SE) and quantitative measures of methylation are presented as mean $\pm \mathrm{SE}$. For the identification of clusters of patients based on quantitative measures of methylation, hierarchical classification, allowing a dendrogram representation, was deduced from the percent of methylation at the 7 cytosines of the four DMRs using euclidean distance and Ward method. Euclidean distances were computed between individuals, then the closest ones were merged into a cluster. Distances between clusters are defined by the Ward formula. Euclidean distance is the square root of the sum of squares of differences between each coordinate of two points. For example, if we have two points $\mathrm{x}$ and $\mathrm{y}$ defined by two coordinates $(\mathrm{x} 1, \mathrm{x} 2)$ and $(\mathrm{y} 1, \mathrm{y} 2)$ respectively, the Euclidean distance between them is $\operatorname{sqrt}\left((\mathrm{x} 1-\mathrm{y} 1)^{\wedge} 2+(\mathrm{x} 2-\mathrm{y} 2)^{\wedge} 2\right)$. Ward distance is exactly the same but it is based on the coordinates of the cluster centroids and is weighted by the cluster sizes. Merging was 
performed until all data were clustered in a single group. The findings were confirmed by a principal component analysis (PCA) (24). PCA is a statistical method that reduces the complexity of the interrelationships among a large number of variables ( 7 cytosines of the four DMRs in this report) to a small number of linear combinations, referred to as principal components. The first principal component is the linear combination of original variables which spreads the data as much as possible. Then, the following components are built so that they also maximize the variance of data while bringing new information with regards to the previous components (orthogonality between successive components). Hence, successive component accounts for decreasing and additive parts of the total variability of data. In our study, the first three components accounted for $93 \%$ of the variability (component 1:72\%, component 2: $13 \%$ and component 3: $8 \%$ ), which renders the study of these three principal components highly sufficient. Because the sample sizes are small, the non-parametric Kruskal Wallis test was used to compare percent of methylation and clinical characteristics between clusters and sub-clusters. The non-parametric multiple comparison test was used to further study the differences between pairs of clusters (25). The Wilcoxon Signed Rank Test was used to compare height and BMI values to the general population reference (hypothetical value $=0$ ). For the correlation between variables, degree of significance and confidence interval are provided. Statistical analyses have been performed using R and Prism softwares (26). 


\section{RESULTS}

Diagnosis of PHP-Ib (Figure 2)

Three subjects (PHP1, 2, 3) carry the 3-kb STX16 deletion (data not shown) and loss of methylation at the A/B DMR of GNAS without additional epigenetic change. The 3-kb STX16 deletion was also found in their mothers. The 3-kb STX16 deletion was not found in the 16 remaining patients. As expected, these patients displayed loss of methylation at the A/B DMR of GNAS and methylation change(s) affecting at least one additional DMR. The quantitative real-time amplification of a target sequence within the NESP exon was similar in the 16 patients affected with spor-PHPIb and in 3 controls than that of one single-copy gene and thus renders unlikely a deletion removing one allele (data not shown). DNAs of the two parents of patients PHP4, 12,13,14, 16 and 17, of the mother of patients PHP9 and 19 and of the siblings of patients PHP16 and 17 were available. None of the parents or siblings had methylation abnormalities at the GNAS locus (combined bisulfite restriction analysis). Altogether, our cohort of PHP-Ib comprises 3 patients affected with AD-PHP-Ib (PHP1-3) and 16 patients affected with sporPHP-Ib (PHP4-17). Results for combined bisulfite restriction analysis from 2 controls, 2 patients affected with AD-PHP-Ib and 4 patients affected with sporPHP-Ib are shown on Figure 2, panels a-d.

Identical diagnosis of AD- and sporPHP-Ib patients comparing quantification of methylation and combined bisulfite restriction analysis (Figure 2 and 3)

In all patients affected with PHP-Ib, percent of methylation at the A/B DMR ranged from 1.4 to $14.9 \%$, a range that was significantly lower than that observed in controls $(42.4$ to $67.4 \%)(\mathrm{p}=1.21 \mathrm{e}-$ 04). No overlap between values measured in PHP-Ib patients and controls was observed. These results indicate that the quantification of methylation at the A/B DMR cytosines provides a valid and specific diagnosis for PHP-Ib (Figures 2 e and 3 b).

We confirmed the accuracy of the quantification by measuring the methylation at the A/B DMR through pyrosequencing (Supplementary data). 
Furthermore, as expected, in patients with AD-PHP-Ib, percent of methylation at the AS, XL and

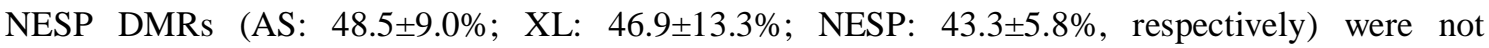
significantly different from that in controls (AS: $46.1 \pm 8.5 \%, p=0,690 ; \mathrm{XL}: 42.8 \pm 4.3 \%, \mathrm{p}=0.930$; NESP: 43.1 $\pm 7.4 \%, \mathrm{p}=0.924$, respectively) (Figures $\mathbf{2} \mathbf{f}-\mathbf{h}$ and $\mathbf{3}$ b).

In patients with sporPHP-Ib, percent of methylation were significantly lower at the AS and XL DMRs (AS: $2.3 \pm 6.6 \%, p=1.15 \mathrm{e}-06$; XL: $18.3 \pm 10.9 \%, \mathrm{p}=4.37 \mathrm{e}-06$ ) and significantly higher at the NESP DMR (79.8 $\pm 17.7 \%, p=1.90 \mathrm{e}-05)$ than those of controls (Figure $2 \mathbf{f}-\mathbf{h})$.

These results indicate that the quantification of methylation at the AS, XL and NESP DMR cytosines provides a reliable diagnosis tool to discriminate between AD-PHPIb with loss of methylation restricted to the DMR A/B and sporPHP-Ib with broad methylation changes.

\section{Classification of patients through the quantification of methylation at the four DMRs of GNAS}

(Figure 3 and Supplementary Figure 3).

Hierarchical classification and PCA using the percent of methylation at the studied cytosines $(n=7)$, first performed comparing controls and PHP-Ib patients, provided three clusters (cluster 1, 2 and 3) that matched the classification in control, AD-PHP-Ib and sporPHP-Ib groups, respectively, established on the basis of the combined bisulfite restriction analysis (Figure 3 and Supplementary Figure 3).

Further analysis of cluster 3 identified three different sub-clusters (designated here as sub-clusters 3.1, 3.2 and 3.3) reflecting three different patterns of methylation and likely, three different types of sporPHP-Ib. For each sub-cluster obtained through PCA, individual percent of methylation at each DMR are shown on Figure $3 \mathbf{~ b}$. Sub-cluster 3.1 is characterized by incomplete methylation changes at AS, XL and NESP DMRs, sub-cluster 3.2 by a methylation at the XL DMR similar to that in controls, whereas patients in sub-cluster 3.3 showed profound epigenetic defects at all DMRs investigated.

\section{Correlation epigenotype-phenotype}

Similar to what we and others have previously reported (8) (9), some patients with sporPHP-Ib display features of Albright osteodystrophy initially described in patients affected with PHP-Ia (Table 1). 
PHP9 presented with infracentrimetric subcutaneous ossification and PHP4, PHP17 and PHP12 with short metacarpals. The distribution of these patients was equal in the 3.1, 3.2 and 3.3 sub-clusters. In patients with sporPHP-Ib, we also observed a distribution of body mass indexes (BMI) towards obesity (mean BMI 1.0 \pm 0.4 SD, p=1.9e-02). The females showed a significantly higher BMI than the males $(2.0 \pm 1.0 \mathrm{SD}$ and $0.1 \pm 0.9 \mathrm{SD}, \mathrm{p}=3.5 \mathrm{e}-03$ respectively). However, the mean height for these patients $(0.6 \pm 0.5 \mathrm{SD})$ does not differ from that of the French reference population $(\mathrm{p}=0.268)(27)$. One patient with AD-PHP-Ib (PHP1) also presented with an increased BMI (Table 1). Interestingly, we found a significant positive correlation between the percent of methylation at the $\mathrm{A} / \mathrm{B}$ DMR and the PTH level at the time of diagnosis (PTH and cytosine AB1: p=5.8e-03, CI 0.20-0.84 ; PTH and cytosine AB2: p=4.0e-04, CI 0.38-0.89) (Figure 4 b) and a negative correlation between PTH and $25 \mathrm{OH}$ vitamin D levels $(\mathrm{p}=1.2 \mathrm{e}-03$, CI $-0.87-0.11)$. PTH levels were not influenced by gender or the age at diagnosis.

Serum calcium, phosphate and PTH levels were similar in the 3.1, 3.2 and 3.3 sub-clusters Figure 4 a. TSH levels were significantly higher in sub-cluster 3.2 compared to sub-clusters 3.1 and $3.3(\mathrm{p}<0.05$ for both comparisons). TSH levels did not correlate with the percent of methylation at the A/B nor the XL DMR. 


\section{DISCUSSION}

We show here, in a series of 19 patients affected with PHP-Ib, diverse patterns of methylation at the GNAS locus. Those results will help to better understand this rare disease. First, we show that sporadic PHP-Ib may have different origins reflected by at least three significantly different patterns of epigenetic abnormalities. Second, we show for the first time through a quantitative method that epigenetic defects are incomplete in some patients. This has been suggested by Liu et al (18) through southern-blotting. This may have important consequences for the diagnosis of the disease and the genetic counselling in patients. Third, we show a positive correlation between PTH level, a hallmark of $\mathrm{G}_{\mathrm{s}} \alpha$ function in the kidney, and quantitative methylation at the A/B DMR measured in blood lymphocytes where $\mathrm{G}_{\mathrm{s}} \alpha$ is not imprinted.

Heterogeneity in the methylation abnormalities at the GNAS locus in patients affected with PHP-Ib, first reported by Liu et al (13), has been since then confirmed by us and others (18) (19) (9) (20). In an effort to better characterize these methylation abnormalities, and because we experienced difficulties in interpretating the results of the combined bisulfite restriction analysis in a subset of patients affected with PHP-Ib, we adapted a quantitative method developed by Thomassin and colleagues (22) to measure the methylation at the four DMRs of GNAS locus. Compared to the southern blot which is considered as the gold-standard quantitative method, the quantification of methylation through realtime PCR i) requires $\approx 20$ times less DNA, ii) can be completed within 4 hours after bisulfite conversion, iii) allows the analysis of twenty samples in one run. However, similarly to methods relying on enzymatic digestion such as southern-blot or methyl-sensitive assays, or on allele-specific hybridization, this method does not allow the analysis of a stretch of cytosines. The latter can be performed through pyrosequencing, sequencing of subcloned PCR products or base-specific cleavage followed by mass spectrometry after amplification from bisulfite treated DNA (28) (9) (29).

Our results show that the quantification of the methylation at the DMRs of GNAS provides a reliable diagnosis of PHP-Ib -i.e. detects loss of methylation at the A/B DMR- and accurately discriminates AD-PHP-Ib from sporPHP-Ib (grouped in clusters 2 and 3, respectively in this report). 
In addition, the quantitative measure of the methylation allowed the identification of three sub-groups of sporPHP-Ib (sub-clusters 3.1, 3.2 and 3.3) based on their pattern of methylation at the four DMRs of GNAS. Our observations raise several hypotheses as regard to the mechanisms involved in these methylation patterns. First, these different patterns may correspond to different causes of methylation defects. So far, all elucidated epigenetic changes at the GNAS locus have been linked to the deletion of an ICE close to or within GNAS (STX16 and NESP-AS, respectively) in AD-PHP-Ib (14) (16) (15) (17). The variable patterns of methylation defects in the GNAS locus that we identified in sporPHP-Ib could be due either to a deletion of different (yet-unknown) GNAS ICEs or to partial deletions of a single ICE. In agreement with such a possibility, in the 15q11-q13 imprinted locus, numerous imprinting center deletions have been identified in patients or generated in mice. Both the phenotype of the patients (and mice) and the methylation pattern of the surrounding region depend on the size of the deletion (30) (31).

Alternatively, besides patients with profound epigenetic changes at the GNAS locus, we identified one group of patients (sub-cluster 3.1) with moderate methylation changes at all DMRs investigated. This pattern would be expected in the presence of somatic mosaicism i.e. the contribution of normal cells and cells with an imprinting defect to the embryo. If an epimutation occurs in the gametes, all cells should be modified leading to drastic changes in the percent of methylation measured by a quantitative method. A range of methylation from 24 to $68 \%$ has been found at the NESP DMR in healthy individuals and attributed to epigenetic mosaicism (32). We observe in patients of sub-clusters 3.1 and 3.2 that the percent of methylation at each analyzed DMR differs within the same patients. In particular, the methylation at the XL DMR appeared less affected compared to the A/B, AS and NESP DMRs. This may suggest that XL is a secondary DMR in which the parental imprint is inconsistently modified whatever the mechanism leading to the observed pattern of methylation, somatic mosaicism (sub-cluster 3.1) or another unknown mechanism (sub-cluster 3.2). Such somatic mosaics of epigenetic defects have also been proposed in the context of several diseases involving loci submitted to parental imprinting. Somatic mosaicism has been found in about one third of the Angelman syndrome with imprinting defects at the SNURF-SNRPN gene and correlated with the severity of the disease (33). In patients with Beckwith-Wiedemann syndrome, Bliek and al. reported ratios of 
unmethylated DMRs ranging from 2.5 to $33 \%$ at KvDMR1 and patterns of both partial and complete hypomethylation at other imprinted loci including GNAS in the same patients (34). In patients with Silver-Russell syndrome, several groups have now reported that the clinical asymmetry is associated with variable degrees of hypomethylation at the $\mathrm{H} 19$ gene in accordance with a post-zygotic error of the imprinting (35) (36). Noteworthy, this mechanism is compatible with the sporadic occurrence of PHP-Ib. Moderate methylation changes could also result from incomplete methylation pattern along the maternal GNAS allele in each cell due to stochastic juxtaposition of methylated and unmethylated cytosines at each DMR. In a previous report, we have shown in two patients affected with sporPHP-Ib (PHP4, sub-cluster 3.1 and PHP17, sub-cluster 3.3) that the methylation of the cytosines is homogeneous along alleles at the four GNAS DMRs (9), thereby rending unlikely stochastic modifications of the methylation as the cause of the incomplete methylation changes that we measured. Demonstration of somatic mosaicism will require clonal analysis of cells derived from affected tissues to show the presence of cells with normal methylation patterns and cells with abnormal methylation patterns.

Uniparental disomy (hetero or isodisomy) has been identified as the cause of diseases of parental imprinting (for example Prader-Willi syndrome (31) or maternal UPD 14 syndrome (37)) and in one patient affected with PHP-Ib (38). Therefore we cannot exclude that, in some patients, PHP-Ib is due to uniparental disomy, especially in patients with the profound epigenetic changes (sub-cluster 3.3 in this report).

PTH levels in absence of treatment for PTH resistance were positively correlated with the percent of methylation at the A/B DMR of GNAS. Although higher PTH levels are intuitively associated with more severe PTH resistance, our findings may suggest that the association of hypocalcemia and mildly elevated PTH reflects more severe PTH resistance than hypocalcemia and highly elevated PTH levels. As if, in the latter case, low expression of $\mathrm{G}_{\mathrm{s}} \alpha$ allows the maintenance of the calcium level and PTH signalling at the cost of highly elevated PTH concentration (hypocalcemia occurs with lower levels of PTH when A/B is completely demethylated and $\mathrm{G}_{\mathrm{s}} \alpha$ not expressed). Differently, the percent of methylation at $\mathrm{A} / \mathrm{B}$-i.e. the degree of $\mathrm{G}_{\mathrm{s}} \alpha$ expression- may indirectly control PTH secretion and/or 
clearance through a feedback loop. This correlation between PTH levels and the methylation at the A/B DMR could be influenced by other factors and should be considered carefully. In fact, PTH levels are prone to environmentally-mediated fluctuations, and we have confirmed in this cohort that PTH levels were influenced by $25 \mathrm{OH}$ vitamin D levels. Noteworthy, this correlation was established with data obtained through different techniques. However, we propose that this correlation between PTH and the degree of methylation at the A/B DMR confirms the clinical relevance of studying the methylation at the GNAS locus in blood lymphocytes where $\mathrm{G}_{\mathrm{s}} \alpha$ is not imprinted.

Comparison of the clinical and biochemical characteristics between sub-clusters of patients with sporPHP-Ib showed that serum TSH levels were lower in sub-clusters 3.1 and 3.3 compared to subcluster 3.2. Despite the low number of patients in each group, which does not allow to draw definitive conclusions, it is interesting that sub-cluster 3.1 and 3.3 are characterized by a more profound loss of methylation at the XL DMR compared to that in sub-cluster 3.2. The loss of methylation at the XL DMR likely allows the biallelic expression of $\mathrm{XL} \alpha_{\mathrm{S}}$ (39), a protein that arises from XL, which shares with $\mathrm{G}_{\mathrm{s}} \alpha$ the ability to generate intracellular cAMP (40) (41). One can speculate that increase in XL $\alpha_{\mathrm{s}}$ expression resulting from lower methylation at the XL DMR may compensate for the $G_{s} \alpha$ deficiency in the thyroid and prevent TSH resistance in patients of sub-clusters 3.1 and 3.3. If this hypothesis is true, then patients affected with AD-PHP-Ib, who have a monoallelic expression of XL should have elevated TSH levels. In the patients reported here, TSH levels were 4.5 (PHP1) and $1.9 \mathrm{mUI} / \mathrm{L}$ (PHP2) (normal range: $0.5-4.5 \mathrm{mUI} / \mathrm{L}$ ). Interestingly, the TSH level in the patient affected with AD-PHP-Ib reported by Mantovani et al is 5.4 (42). Altogether, these results indicate that some phenotypic characteristics of patients may depend on the pattern of methylation at the GNAS locus, yet need to be confirmed in a larger cohort of patients.

By the precise characterization of the methylation defects at the GNAS locus in a cohort of PHP-Ib patients with PHP-Ib, we have identified different subtypes of PHP-Ib compatible with different causes of the disease, including somatic mosaicism. 


\section{ACKNOWLEDGMENTS}

We thank the affected individuals and their families for their collaboration. We thank Gilles Grange for his help in the quantitative assessment of the methylation. We thank Pierre-François Bougnères and Bernard Grandchamp for their constant support and helpful discussions.

This work was supported by INSERM funding. S.M-M. was supported by a fellowship from the Paris Descartes University.

The authors declare no conflict of interest.

\section{COPYRIGHT licence statement}

The Corresponding Author has the right to grant on behalf of all authors and does grant on behalf of all authors, an exclusive licence (or non-exclusive for government employees) on a worldwide basis to the BMJ Publishing Group Ltd, and its Licensees to permit this article (if accepted) to be published in the Journal of Medical Genetics and any other BMJPGL products and to exploit all subsidiary rights, as set out in our licence 


\section{LEGENDS}

Figure 1: Schematic drawing of the GNAS locus and its 5' boundary including the STX16 gene. The GNAS locus is scaled, based on HG18. The position of the 7 cytosines is indicated by the vertical lines and the numbers. The four DMRs studied in this report are represented below the genomic line by black boxes (+ or methylated) or white boxes (- or unmethylated) on the paternal (Pat) or maternal (Mat) allele (2). CpG islands identified by the Genome Browser software are shown on the lower horizontal line. Exons are indicated as black rectangles, repeated sequences as stripped triangles, STX16 and NESP-AS deletions as brackets, allelic origin of transcription as broken arrows on the paternal (Pat) or maternal (Mat) allele.

Figure 2: Methylation analysis at the four DMRs of GNAS. (a.b.c.d): Electrophoresis on an agarose gel after combined bisulfite restriction analysis of genomic DNA at the DMRs A/B, AS, XL and NESP in 2 control individuals (CTRL1, CTRL2) and 6 patients affected with PHP-Ib. At the A/B DMR: in all PHP-Ib patients the lower (i.e. methylated or $\mathrm{CH}_{3}$ ) band is completely or nearly completely absent indicating a loss of methylation. At the AS and XL DMRs: in patients PHP17, PHP18, PHP7 and PHP4, the lower (i.e. methylated) band is completely or nearly completely absent indicating a loss of methylation, whereas it is present in PHP1, PHP2, CTRL1 and CTRL 2, indicating an hemi-methylation. At the NESP DMR: in patients PHP17, PHP18, PHP7 and PHP4, the upper (i.e. unmethylated or un- $\mathrm{CH}_{3}$ ) band is faint indicating a partial gain of methylation, whereas it is similar to controls in PHP1 and PHP2, indicating an hemi-methylation. (e.f.g.h): In the same individuals, percent of methylation $\left(\% \mathrm{CH}_{3}\right)$ measured at one representative cytosine of each DMR $(\mathrm{AB} 2$ for $\mathrm{A} / \mathrm{B}$, AS2 for AS, XL1 for XL and NE for NESP) are represented as bar graphs.

Figure 3: Identification of different subtypes of PHP-Ib. a. Cluster dendrogram obtained after quantification of the methylation at 7 cytosines belonging to four DMRs of GNAS. As exemplified by PCA (see methods and Supplementary Figure 3), three main clusters were identified: cluster 1 which includes all control individuals, cluster 2 which includes the three AD-PHP-Ib patients, and a larger 
cluster 3 including all patients affected with sporPHP-Ib. The latter can be further divided in three sub-clusters: 3.1, 3.2 and 3.3. b and c. For each cluster and sub-cluster obtained through PCA, individual values for percent of methylation are shown (cytosine AB2 for A/B, AS2 for AS, XL1 for $\mathrm{XL}$ and NE for NESP). The mean for each group is indicated by the horizontal line, and significative differences between clusters ( $\mathrm{p}$ value $<0.05$ ) are summarized in the table for each DMR.

Figure 4: Comparison between epigenotypes and phenotypes. a. Box and whisker plots of hormonal resistance markers in the patients of sub-clusters 3.1, 3.2 and 3.3. The shaded area indicates the normal range. *: p<0.05 compared to values in sub-clusters 3.1 and 3.3. b. Significant correlation between the percent of methylation at the A/B DMR and PTH levels at the time of diagnosis. Both correlations with cytosines $\mathrm{AB} 1$ and $\mathrm{AB} 2$ are shown.

\section{Supplementary Figure 1: Quantification of the methylation adapted from Thomassin and}

colleagues (22). A. Sequences of the PCR products amplified for the analysis of the A/B, AS, XL and NESP DMRs. Bold and underlined cytosines were studied using the quantitative method, whereas bold and italic cytosines were studied using combined bisulfite restriction analysis. B. Linear regression between theoretical and measured methylation at the A/B DMR (Cytosine AB1, $\mathrm{r}^{2}=0.98$, $\mathrm{p}<1.0 \mathrm{e}-04)$. C. Schematic drawing of the PCR amplification of a differentially methylated region (the unmethylated cytosine on the paternal allele was previously converted by bisulfite treatment in uracile then in thymine after the first round of amplification) using non discriminative (black arrows) and discriminative (red-lozenge arrow) primers. The amplification and melting curves after amplification of a differentially methylated DNA fragment show the delay in the discriminative (D) compared to the non-discriminative amplification (ND). The melting curves confirm the presence of two different PCR products (shown on the right panel) in the same reaction.

Supplementary Figure 2: Correlations between percent of methylation measured at cytosines AB1 and AB2, XL1 and XL2, AS1 and AS2. Degree of significance (p), confidence interval (CI) and r squared are provided. 
Supplementary Figure 3: Biplots representation of PCA using the quantification of methylation at the four DMR of GNAS (seven cytosines). A biplot is the projection of patients and controls onto two distinct principal components. Variables (quantification of methylation at each cytosine) are shown by red arrows; the length of the arrow indicates the weight of each variable ; the opposite direction of the NESP cytosine versus the other cytosines illustrates gain versus loss of methylation. The black rectangles delineate clusters and sub-clusters based on the hierarchical classification. In biplot a (component 1 and 2), controls and patients are clearly separated based on quantification of methylation at the A/B cytosines. Within patients, AD-PHP-Ib and sporPHP-Ib are distinguished by the methylation at the other DMRs. In biplot $b$ (component 1 and 3), patients with sporPHP-Ib are clearly separated from controls and AD-PHP-Ib. In this group, three sub-clusters of patients can be identified based on the quantification at NESP, XL and AS cytosines. 
Table 1: Clinical and biochemical characteristics at diagnosis of the 19 patients affected with PHP-Ib, either AD-PHP-Ib with STX16 deletion and loss of methylation restricted to the A/B DMR of GNAS, or sporPHP-Ib with broad GNAS methylation changes. M: male, F: female; BMI: body mass index; AHO: Albright hereditary osteodystrophy; BM: brachydactyly; SCO: subcutaneous ossification; na: not available; no: absence of obvious features of AHO. Normal range for calcemia is 2.25-2.60 mM, phosphatemia: 1-1.8 mM, PTH: $10-60 \mathrm{pg} / \mathrm{ml}, 25-\mathrm{OH}$ vitamin D (25-OHD): $15-60 \mathrm{ng} / \mathrm{ml}$ and TSH: 0.5-4.5 mUI/L.

\begin{tabular}{|c|c|c|c|c|c|c|c|c|c|c|c|c|c|c|c|}
\hline $\begin{array}{l}\text { Code } \\
\text { Patient }\end{array}$ & Disease & $\begin{array}{c}\text { Gender } \\
\text { (M/F) }\end{array}$ & $\begin{array}{c}\text { Age at } \\
\text { diagnosis } \\
\text { (years) }\end{array}$ & Height (SD) & BMI $\left(\mathrm{kg} / \mathrm{m}^{2}\right)$ & BMI & $\begin{array}{c}\text { Number of } \\
\text { family } \\
\text { members } \\
\text { studied }\end{array}$ & $\begin{array}{c}\text { Number of } \\
\text { family } \\
\text { members } \\
\text { affected }\end{array}$ & $\begin{array}{c}\text { AHO } \\
\text { features }\end{array}$ & $\begin{array}{l}\text { Calcemia } \\
\text { (mM) }\end{array}$ & $\begin{array}{l}\text { Phosphatemia } \\
\text { (mM) }\end{array}$ & PTH (pg/ml) & TSH (mUl/L) & $\begin{array}{l}\text { 25-OHD } \\
\text { (ng/mL) }\end{array}$ & $\begin{array}{l}\text { Cluster and } \\
\text { sub-cluster }\end{array}$ \\
\hline PHP1 & AD-PHP-Ib & M & 9.0 & -0.4 & 24.1 & 0.3 & 6 & 2 & no & 1.7 & 2.8 & na & 4.5 & 12.0 & 2 \\
\hline PHP2 & AD-PHP-Ib & $\mathrm{F}$ & 15.3 & na & na & na & na & - & no & 1.4 & 2.6 & 890 & 1.9 & 6.0 & 2 \\
\hline PHP3 & AD-PHP-Ib & $\mathrm{M}$ & 14.2 & na & na & na & 2 & 0 & no & 1.3 & 3.0 & 197 & na & 48.0 & 2 \\
\hline PHP4 & SporPHP-lb & $\mathrm{F}$ & 10.0 & 0.3 & 27.5 & 2.2 & 2 & 0 & BM & 1.4 & 2.2 & 169 & 1.0 & 21.0 & 3.1 \\
\hline PHP5 & SporPHP-Ib & M & 14.5 & 0.7 & 23.1 & 1.6 & na & - & no & 1.1 & 2.5 & 259 & 5.3 & na & 3.1 \\
\hline PHP6 & SporPHP-lb & M & 11.0 & 5.4 & 27.7 & 3.7 & na & - & no & 1.7 & 2.1 & 551 & 2.6 & 25.0 & 3.1 \\
\hline PHP7 & SporPHP-lb & M & 7.0 & 3.4 & 19.9 & 2.5 & na & - & no & 1.0 & 3.0 & 583 & 2.0 & 9.0 & 3.1 \\
\hline PHP8 & SporPHP-lb & $M$ & 7.0 & -0.2 & 17.8 & 0.0 & na & - & no & 1.8 & 2.2 & 386 & 2.2 & na & 3.1 \\
\hline PHP9 & SporPHP-lb & $\mathrm{F}$ & 8.0 & 1.6 & 23.1 & 1.4 & 1 & 0 & $\mathrm{sco}$ & 1.2 & 2.4 & 572 & 6.5 & 9.0 & 3.2 \\
\hline PHP10 & SporPHP-Ib & $\mathrm{F}$ & 26.0 & -2.7 & 26.2 & 1.2 & na & - & no & 1.3 & 1.9 & 359 & 5.5 & 25.6 & 3.2 \\
\hline PHP11 & SporPHP-lb & $\mathrm{F}$ & 16.0 & 0.1 & 23.0 & 0.8 & na & - & no & 1.7 & 2.0 & 195 & 10.1 & 32.8 & 3.2 \\
\hline PHP12 & SporPHP-Ib & $\mathrm{F}$ & 26.0 & 0.1 & 24.3 & 0.6 & 2 & 0 & BM & 1.8 & 1.2 & 807 & 2.7 & na & 3.3 \\
\hline PHP13 & SporPHP-Ib & $M$ & 14.0 & 0.6 & 16.8 & -1.6 & 2 & 0 & no & 1.2 & 2.4 & 603 & 3.8 & na & 3.3 \\
\hline PHP14 & SporPHP-Ib & $M$ & 7.0 & 0.0 & 24.0 & 0.4 & 2 & 0 & no & 2.2 & 2.2 & 191 & 3.1 & 22.0 & 3.3 \\
\hline PHP15 & SporPHP-Ib & $M$ & 16.0 & -1.9 & 19.4 & -0.5 & na & - & no & 1.2 & 2.4 & 788 & 3.5 & 8 & 3.3 \\
\hline PHP16 & SporPHP-Ib & $\mathrm{F}$ & 10.5 & 3.2 & 40.9 & 4.0 & 4 & 0 & no & 1.5 & 2.2 & 126 & 3.6 & 25.5 & 3.3 \\
\hline PHP17 & SporPHP-Ib & M & 11.0 & -0.3 & 18.4 & -1.1 & 5 & 0 & BM & 1.4 & 2.2 & 185 & 6.6 & 11.0 & 3.3 \\
\hline PHP18 & SporPHP-Ib & M & 10.4 & -1.6 & 16.8 & 0.1 & na & 0 & no & 1.3 & 3.2 & 410 & 2.5 & 9.0 & 3.3 \\
\hline PHP19 & SporPHP-Ib & $\mathrm{M}$ & 13.0 & 0.7 & 23.9 & 1.7 & 1 & 0 & no & 1.3 & 3.3 & 232 & 2.4 & na & 3.3 \\
\hline
\end{tabular}




\section{REFERENCES}

1. Ideraabdullah F, Vigneau S, Bartolomei MS 2008 Genomic imprinting mechanisms in mammals. Mutation Research 647:77-85

2. Hayward BE, Moran V, Strain L, Bonthron DT 1998 Bidirectional imprinting of a single gene: GNAS1 encodes maternally, paternally, and biallelically derived proteins. Proc Natl Acad Sci USA 95:15475-15480

3. Hayward B, Bonthron D 2000 An imprinted antisense transcript at the human GNAS1 locus. Hum Mol Genet 9:835-841

4. Yu S, Yu D, Lee E, Eckhaus M, Lee R, Corria Z, Accili D, Westphal H, Weinstein LS 1998 Variable and tissue-specific hormone resistance in heterotrimeric $\mathrm{G}_{\mathrm{s}}$ protein $\alpha$-subunit $\left(\mathrm{G}_{\mathrm{s}} \alpha\right)$ knockout mice is due to tissue-specific imprinting of the $\mathrm{G}_{\mathrm{s}} \alpha$ gene. Proc Natl Acad Sci USA 95:8715-8720

5. Hayward B, Barlier A, Korbonits M, Grossman A, Jacquet P, Enjalbert A, Bonthron D 2001 Imprinting of the G(s) $\alpha$ gene GNAS1 in the pathogenesis of acromegaly. J Clin Invest 107:R31-36

6. Mantovani G, Ballare E, Giammona E, Beck-Peccoz P, Spada A 2002 The Gs $\alpha$ gene: predominant maternal origin of transcription in human thyroid gland and gonads. J Clin Endocrinol Metab 87:4736-4740

7. Liu J, Erlichman B, Weinstein LS 2003 The stimulatory G protein $\alpha$-subunit Gs $\alpha$ is imprinted in human thyroid glands: implications for thyroid function in pseudohypoparathyroidism types 1a and 1b. J Clin Endocrinol Metabol 88:4336-4341

8. de Nanclares GP, Fernandez-Rebollo E, Santin I, Garcia-Cuartero B, Gaztambide S, Menendez E, Morales MJ, Pombo M, Bilbao JR, Barros F, Zazo N, Ahrens W, Juppner H, Hiort O, Castano L, Bastepe M 2007 Epigenetic defects of GNAS in patients with pseudohypoparathyroidism and mild features of Albright's hereditary osteodystrophy. J Clin Endocrinol Metab 92:2370-2373

9. Mariot V, Maupetit-Mehouas S, Sinding C, Kottler ML, Linglart A 2008 A maternal epimutation of GNAS leads to Albright osteodystrophy and parathyroid hormone resistance. J Clin Endocrinol Metab 93:661-665

10. Mantovani G, de Sanctis L, Barbieri AM, Elli FM, Bollati V, Vaira V, Labarile P, Bondioni S, Peverelli E, Lania AG, Beck-Peccoz P, Spada A 2010 Pseudohypoparathyroidism and GNAS epigenetic defects: clinical evaluation of albright hereditary osteodystrophy and molecular analysis in 40 patients. J Clin Endocrinol Metab 95:651-658

11. Weinstein LS, Gejman PV, Friedman E, Kadowaki T, Collins RM, Gershon ES, Spiegel AM 1990 Mutations of the Gs $\alpha$-subunit gene in Albright hereditary osteodystrophy detected by denaturing gradient gel electrophoresis. Proc Natl Acad Sci USA 87:8287-8290

12. Linglart A, Carel JC, Garabedian M, Le T, Mallet E, Kottler ML 2002 GNAS1 Lesions in Pseudohypoparathyroidism Ia and Ic: Genotype Phenotype Relationship and Evidence of the Maternal Transmission of the Hormonal Resistance. J Clin Endocrinol Metab 87:189-197

13. Liu J, Litman D, Rosenberg M, Yu S, Biesecker L, Weinstein L 2000 A GNAS1 imprinting defect in pseudohypoparathyroidism type Ib. J Clin Invest 106:1167-1174

14. Bastepe M, Fröhlich LF, Hendy GN, Indridason OS, Josse RG, Koshiyama H, Korkko J, Nakamoto JM, Rosenbloom AL, Slyper AH, Sugimoto T, Tsatsoulis A, Crawford JD, Jüppner H 2003 Autosomal dominant pseudohypoparathyroidism type Ib is associated with a heterozygous microdeletion that likely disrupts a putative imprinting control element of GNAS. J Clin Invest 112:1255-1263

15. Linglart A, Gensure RC, Olney RC, Juppner H, Bastepe M 2005 A novel STX16 deletion in autosomal dominant pseudohypoparathyroidism type Ib redefines the boundaries of a cisacting imprinting control element of GNAS. Am J Hum Genet 76:804-814

16. Bastepe M, Fröhlich LF, Linglart A, Abu-Zahra HS, Tojo K, Ward LM, Jüppner H 2005 Deletion of the NESP55 differentially methylated region causes loss of maternal GNAS imprints and pseudohypoparathyroidism type Ib. Nat Genet 37:25-27 
17. Chillambhi S, Turan S, Hwang DY, Chen HC, Juppner H, Bastepe M 2010 Deletion of the Noncoding GNAS Antisense Transcript Causes Pseudohypoparathyroidism Type Ib and Biparental Defects of GNAS Methylation in cis. J Clin Endocrinol Metab

18. Liu J, Nealon JG, Weinstein LS 2005 Distinct patterns of abnormal GNAS imprinting in familial and sporadic pseudohypoparathyroidism type IB. Hum Mol Genet 14:95-102

19. Linglart A, Bastepe M, Juppner H 2007 Similar clinical and laboratory findings in patients with symptomatic autosomal dominant and sporadic pseudohypoparathyroidism type $\mathrm{Ib}$ despite different epigenetic changes at the GNAS locus. Clin Endocrinol (Oxf) 67:822-831

20. Jan de Beur S, Ding C, Germain-Lee E, Cho J, Maret A, Levine MA 2003 Discordance between genetic and epigenetic defects in pseudohypoparathyroidism type $1 \mathrm{~b}$ revealed by inconsistent loss of maternal imprinting at GNAS1. Am J Hum Genet 73:314-322

21. Soufir N, Gerard B, Portela M, Brice A, Liboutet M, Saiag P, Descamps V, Kerob D, Wolkenstein P, Gorin I, Lebbe C, Dupin N, Crickx B, Basset-Seguin N, Grandchamp B 2006 PTCH mutations and deletions in patients with typical nevoid basal cell carcinoma syndrome and in patients with a suspected genetic predisposition to basal cell carcinoma: a French study. Br J Cancer 95:548-553

22. Thomassin H, Kress C, Grange T 2004 MethylQuant: a sensitive method for quantifying methylation of specific cytosines within the genome. Nucleic Acids Res 32:e168

23. Olek A, Oswald J, Walter J 1996 A modified and improved method for bisulphite based cytosine methylation analysis. Nucleic Acids Res 24:5064-5066

24. Lebart L, Morineau A, Piron M 2004 Statistiques exploratoires multidimensionnelles

25. Conover. WJ 1980 Practical non-parametric statistics 2nd edition

26. Team RDC 2009 R: A Language and Environment for

Statistical Computing. In: R Foundation for Statistical Computing. Vienna

27. Sempé M, G. GP, P. PR 1979 Auxologie: méthodes et séquences. Paris

28. Tost J, Gut IG 2007 DNA methylation analysis by pyrosequencing. Nat Protoc 2:2265-2275

29. Ehrich M, Nelson MR, Stanssens P, Zabeau M, Liloglou T, Xinarianos G, Cantor CR, Field JK, van den Boom D 2005 Quantitative high-throughput analysis of DNA methylation patterns by base-specific cleavage and mass spectrometry. Proc Natl Acad Sci U S A 102:15785-15790

30. Buiting K, Saitoh S, Gross S, Dittrich B, Schwartz S, Nicholls RD, Horsthemke B 1995 Inherited microdeletions in the Angelman and Prader-Willi syndromes define an imprinting centre on human chromosome 15. Nat Genet 9:395-400

31. Horsthemke B, Wagstaff J 2008 Mechanisms of imprinting of the Prader-Willi/Angelman region. Am J Med Genet A 146A:2041-2052

32. Schneider E, Pliushch G, El Hajj N, Galetzka D, Puhl A, Schorsch M, Frauenknecht K, Riepert T, Tresch A, Muller AM, Coerdt W, Zechner U, Haaf T 2010 Spatial, temporal and interindividual epigenetic variation of functionally important DNA methylation patterns. Nucleic Acids Res

33. Nazlican H, Zeschnigk M, Claussen U, Michel S, Boehringer S, Gillessen-Kaesbach G, Buiting K, Horsthemke B 2004 Somatic mosaicism in patients with Angelman syndrome and an imprinting defect. Hum Mol Genet 13:2547-2555

34. Bliek J, Verde G, Callaway J, Maas SM, De Crescenzo A, Sparago A, Cerrato F, Russo S, Ferraiuolo S, Rinaldi MM, Fischetto R, Lalatta F, Giordano L, Ferrari P, Cubellis MV, Larizza L, Temple IK, Mannens MM, Mackay DJ, Riccio A 2009 Hypomethylation at multiple maternally methylated imprinted regions including PLAGL1 and GNAS loci in Beckwith-Wiedemann syndrome. Eur J Hum Genet 17:611-619

35. Bruce S, Hannula-Jouppi K, Peltonen J, Kere J, Lipsanen-Nyman M 2009 Clinically distinct epigenetic subgroups in Silver-Russell syndrome: the degree of H19 hypomethylation associates with phenotype severity and genital and skeletal anomalies. J Clin Endocrinol Metab 94:579-587

36. Azzi S, Rossignol S, Steunou V, Sas T, Thibaud N, Danton F, Le Jule M, Heinrichs C, Cabrol S, Gicquel C, Le Bouc Y, Netchine I 2009 Multilocus methylation analysis in a large cohort of 11p15-related foetal growth disorders (Russell Silver and Beckwith Wiedemann 
syndromes) reveals simultaneous loss of methylation at paternal and maternal imprinted loci. Hum Mol Genet 18:4724-4733

37. Fokstuen S, Ginsburg C, Zachmann M, Schinzel A 1999 Maternal uniparental disomy 14 as a cause of intrauterine growth retardation and early onset of puberty. J Pediatr 134:689-695

38. Bastepe M, Lane AH, Juppner H 2001 Paternal uniparental isodisomy of chromosome 20q-and the resulting changes in GNAS1 methylation--as a plausible cause of pseudohypoparathyroidism. Am J Hum Genet 68:1283-1289

39. Freson K, Izzi B, Labarque V, Van Helvoirt M, Thys C, Wittevrongel C, Bex M, Bouillon R, Godefroid N, Proesmans W, de Zegher F, Jaeken J, Van Geet C 2008 GNAS defects identified by stimulatory $\mathrm{G}$ protein alpha-subunit signalling studies in platelets. J Clin Endocrinol Metab 93:4851-4859

40. Bastepe M, Gunes Y, Perez-Villamil B, Hunzelman J, Weinstein LS, Jüppner H 2002 Receptor-mediated adenylyl cyclase activation through XLos, the extra-large variant of the stimulatory G protein $\alpha$-subunit. Mol Endocrinol 16:1912-1919

41. Linglart A, Mahon MJ, Kerachian MA, Berlach DM, Hendy GN, Juppner H, Bastepe M 2006 Coding GNAS mutations leading to hormone resistance impair in vitro agonist- and cholera toxin-induced adenosine cyclic $3{ }^{\prime}, 5^{\prime}$-monophosphate formation mediated by human XLalphas. Endocrinology 147:2253-2262

42. Mantovani G, Bondioni S, Linglart A, Maghnie M, Cisternino M, Corbetta S, Lania AG, Beck-Peccoz P, Spada A 2007 Genetic analysis and evaluation of resistance to thyrotropin and growth hormone-releasing hormone in pseudohypoparathyroidism type Ib. J Clin Endocrinol Metab 92:3738-3742 


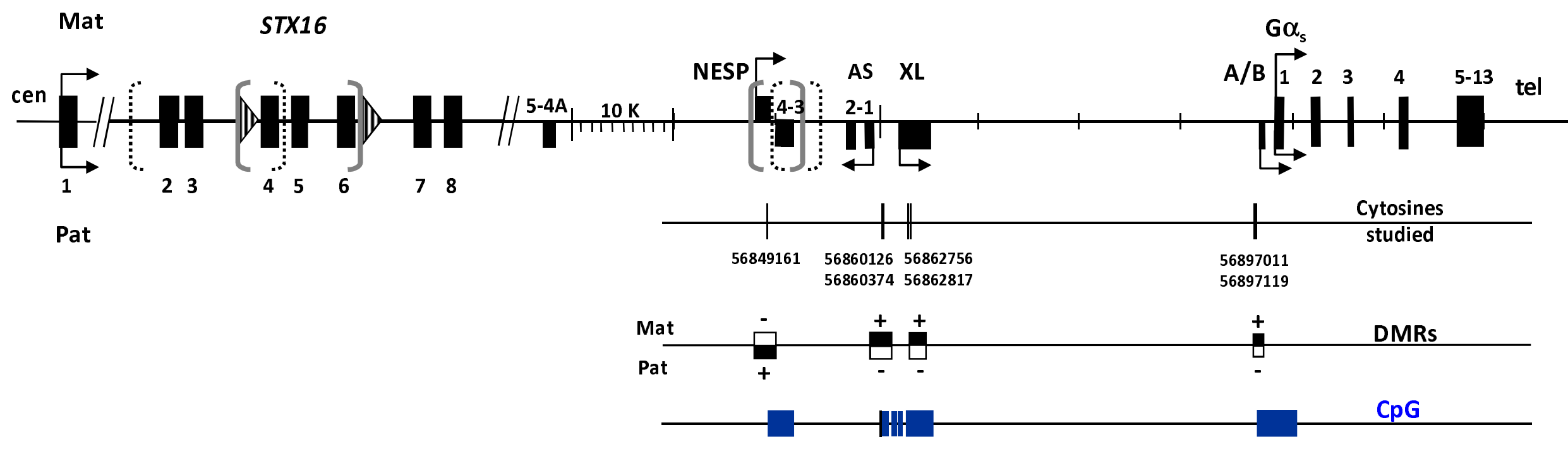

Figure 1 

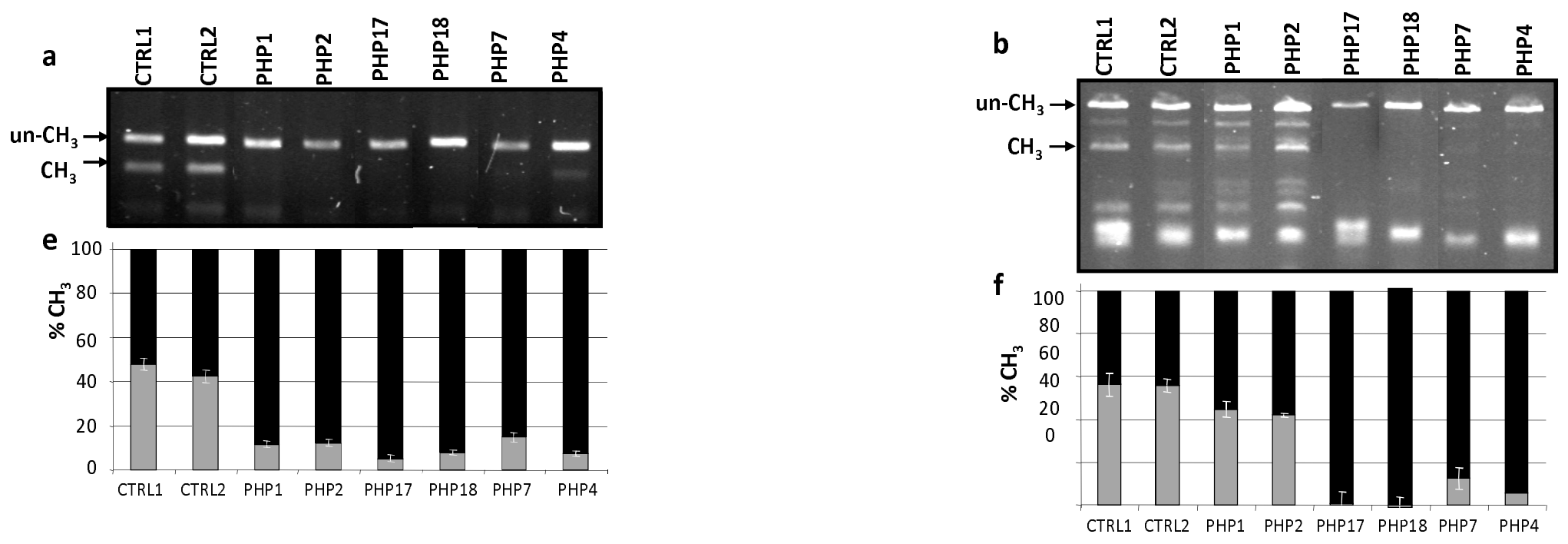

XL

NESP
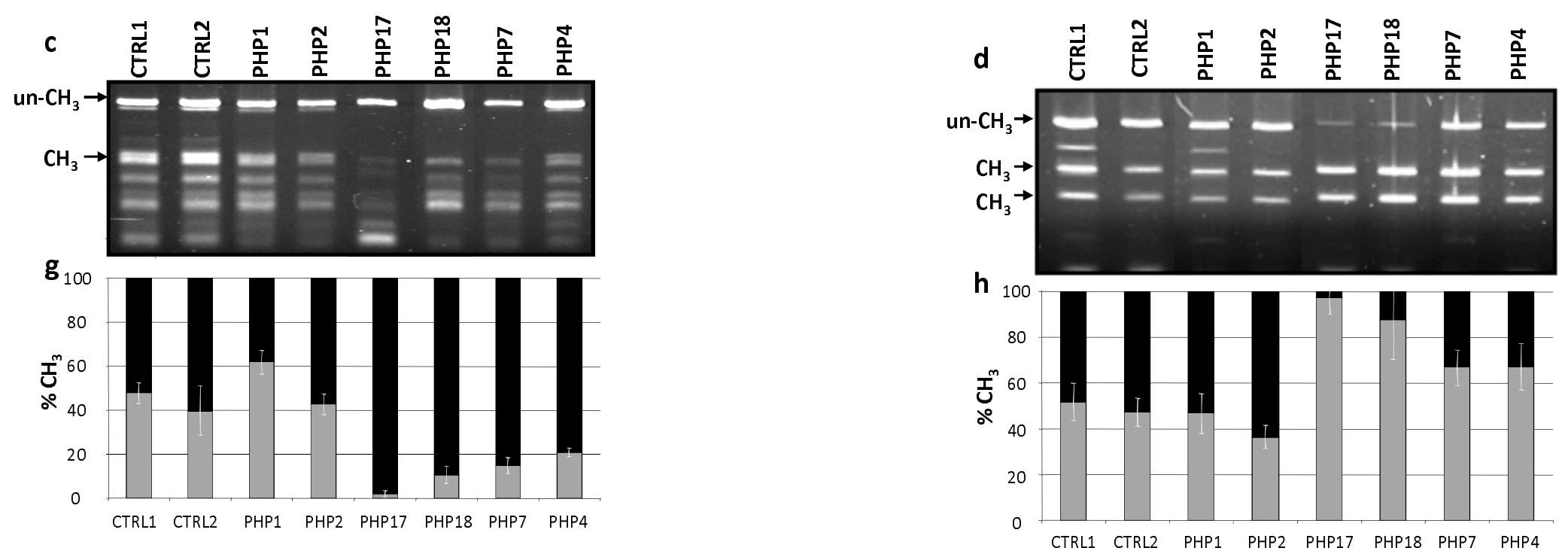

Figure 2 

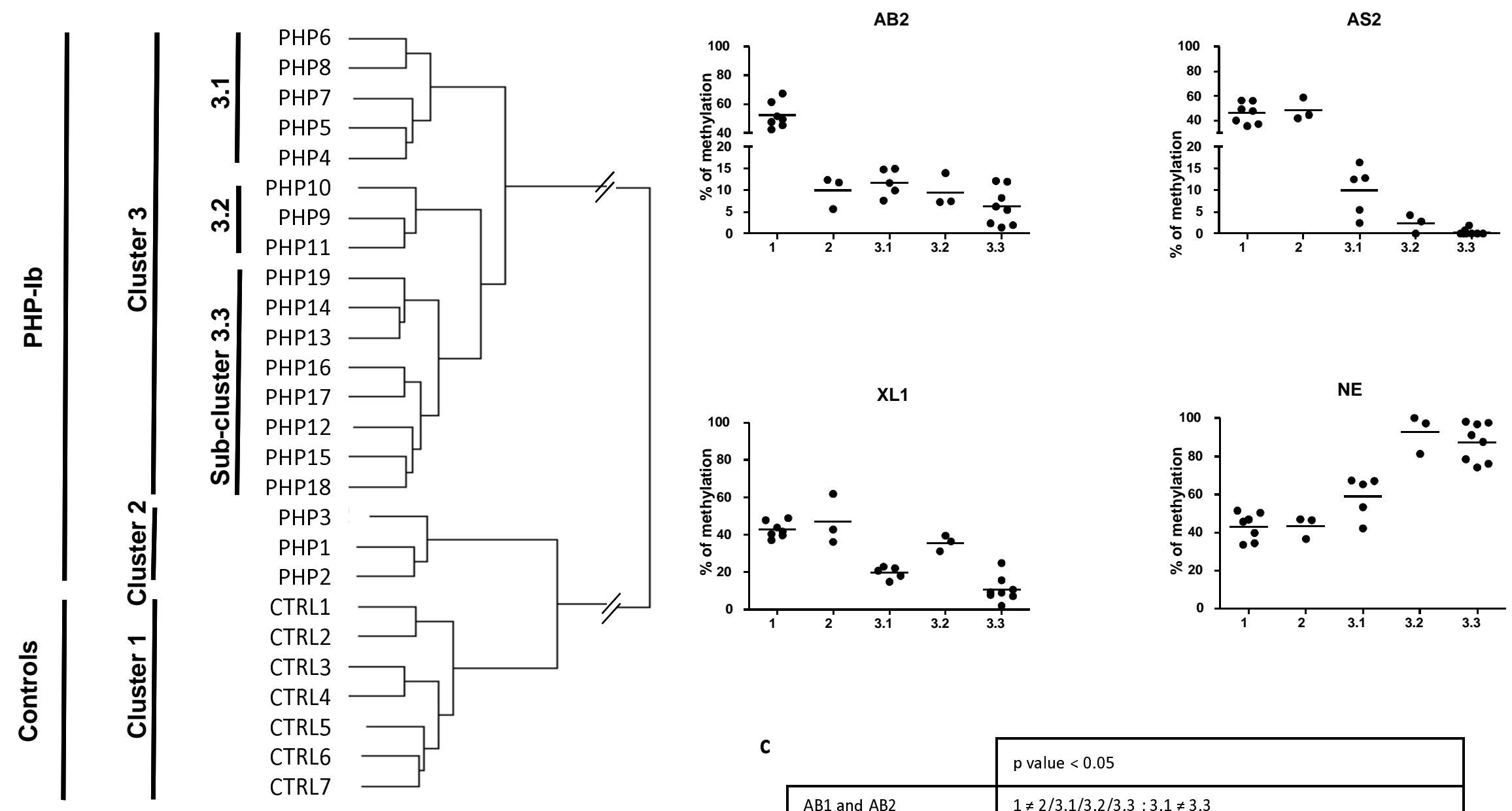

\begin{tabular}{|c|c|}
\hline & $\mathrm{p}$ value $<0.05$ \\
\hline$A B 1$ and $A B 2$ & $1 \neq 2 / 3.1 / 3.2 / 3.3 ; 3.1 \neq 3.3$ \\
\hline AS1 and AS2 & $1 \neq 3.1 / 3.2 / 3.3 ; 2 \neq 3.1 / 3.2 / 3.3 ; 3.1 \neq 3.3 ; 3.2 \neq 3.3$ \\
\hline$X L 1$ and $X L 2$ & $1 \neq 3.1 / 3.2 / 3.3 ; 2 \neq 3.1 / 3.3 ; 3.1 \neq 3.3 ; 3.2 \neq 3.3$ \\
\hline NE & $1 \neq 3.1 / 3.2 / 3.3 ; 2 \neq 3.1 / 3.2 / 3.3 ; 3.1 \neq 3.2 / 3.3$ \\
\hline
\end{tabular}

\section{Figure 3}



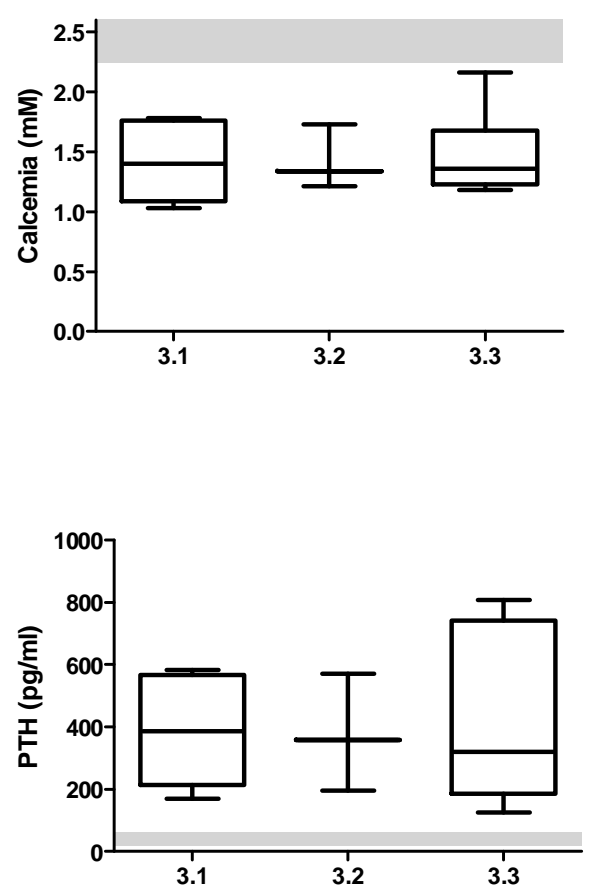

b
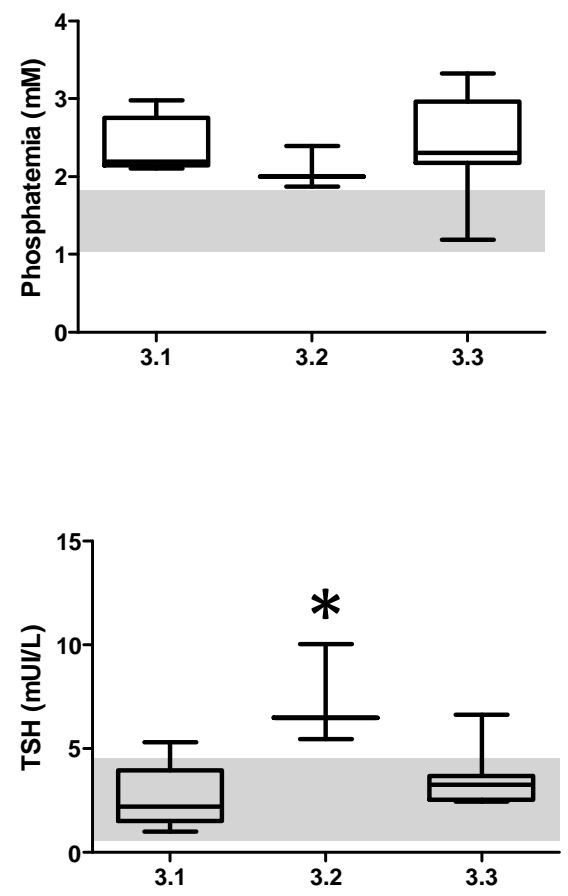

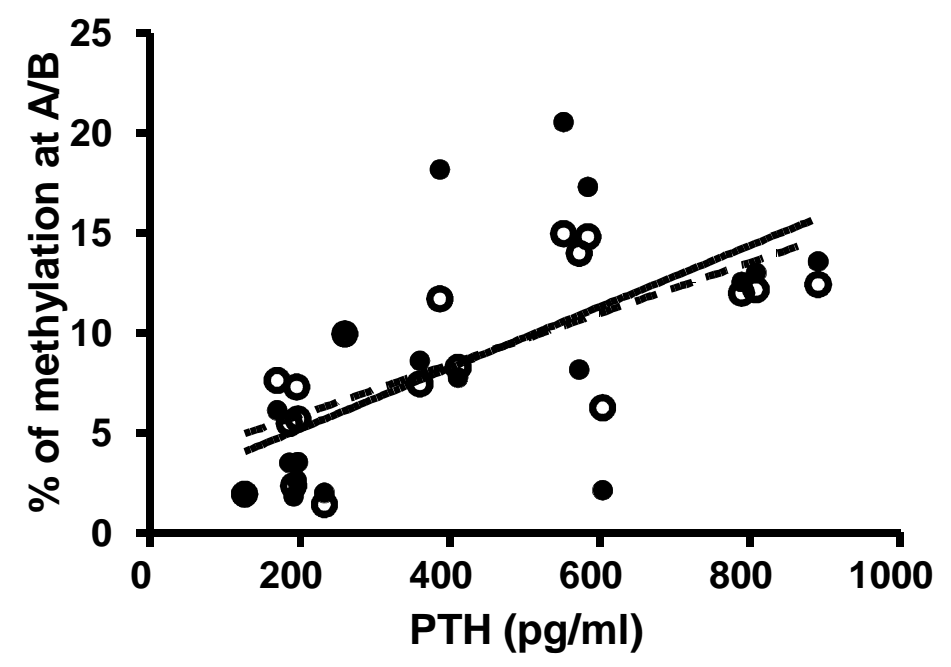

Figure 4 